\title{
Networking Behavior in Thin Film and Nanostructure Growth Dynamics
}

\author{
Murat Yuksel \\ University of Nevada - Reno \\ Reno, NV 89557, USA \\ yuksem@cse.unr.edu
}

\author{
Tansel Karabacak \\ University of Arkansas \\ Little Rock, AR 72204, USA. \\ txkarabacak@ualr.edu
}

\author{
Hasan Guclu \\ Los Alamos National Lab \\ Los Alamos, NM 87545, USA. \\ guclu@lanl.gov
}

\begin{abstract}
Thin film coatings have been essential in development of several micro and nano-scale devices. To realize thin film coatings various deposition techniques are employed, each yielding surface morphologies with different characteristics of interest. Therefore, understanding and control of the surface growth is of great interest. In this paper, we devise a novel network-based modeling of the growth dynamics of such thin films and nanostructures. We specifically map dynamic steps taking place during the growth to components (e.g., nodes, links) of a corresponding network. We present initial results showing that this network-based modeling approach to the growth dynamics can simplify our understanding of the fundamental physical dynamics such as shadowing and re-emission effects.
\end{abstract}

\section{Categories and Subject Descriptors}

H.1 [Models and Principles]: Miscellaneous; I.6 [Simulation and Modeling]: Model Development; C.2.1 [ComputerCommunication Networks]: Network Architecture and Design-network topology, network communications

\section{INTRODUCTION}

Thin film coatings have been the essential components of various devices in industries including microelectronics, optoelectronics, detectors, sensors, micro-electro-mechanical systems (MEMS), and more recently nano-electro-mechanical systems (NEMS). These coatings have thicknesses typically in the nano- to micro-scales and are grown using vacuum deposition techniques [2]. Thin film surface morphology controls many important physical and chemical properties of the films. It is therefore of great interest to understand and control the development of the surface morphology during thin film growth.

Commonly employed deposition techniques are thermal evaporation, sputter deposition, chemical vapor deposition $(C V D)$, and oblique angle deposition. Different than others, oblique angle deposition technique [7] is typically used

Nano-Net 2007 September 24-26, 2007, Catania, Italy.

Copyright 2007 ICST ISBN 978-963-9799-10-3

DOI 10.4108/ICST.NANONET2007.2008 for the growth of nanostructured arrays of rods and springs through a physical self-assembly process. In many applications, it is often desired to have atomically flat thin film surfaces. However, in almost all of the deposition techniques mentioned above, the surface morphology generates a growth front roughness. The formation of growth front is a complex phenomenon and very often occurs far from equilibrium. When atoms are deposited on a surface, atoms do not arrive at the surface at the same time uniformly across the surface. This random fluctuation, or noise, which is inherent in the process, may create the surface roughness. The noise competes with surface smoothening processes, such as surface diffusion (hopping), to form a rough morphology if the experiment is performed at either a sufficiently low temperature or a high growth rate.

A conventional statistical mechanics treatment cannot be used to describe this complex phenomenon. About two decades ago, a dynamic scaling approach [6,5] was pro posed to describe the morphological evolution of a growth front. Since then, numerous modeling and experimental works have been reported based on this dynamic scaling analysis [2]. On the other hand, there has been a significant discrepancy among the predictions of these growth models and the experimental results published [9]. Briefly, theoretical predictions of growth models in dynamic scaling theory basically fall into two categories. One involves various surface smoothing effects, such as surface diffusion. The other category involves the shadowing effect (which originates from the preferential deposition of obliquely incident atoms on higher surface points and always occurs in sputtering and CVD) during growth. However, experimentally reported values of growth exponent (which measures how fast the root-mean-square roughness of the surface evolves as a function of time according to a power-law relation) are far from agreement with the predictions of these growth models. Especially, sputtering and CVD techniques are observed to produce morphologies ranging from very small to very large growth exponent values.

Understanding the thin film and nanostructure growth dynamics under the above-mentioned deposition techniques has been of high importance. There have been several studies revealing fundamental dynamic effects (e.g., shadowing, re-emission, surface-diffusion, and noise effects) taking place during the growth process. Studies towards explaining the growth dynamics have been partly successful and only the simulation-based studies were able to include all these effects. In this paper, we devise a novel network-based modeling approach to better understand the growth dynamics. 


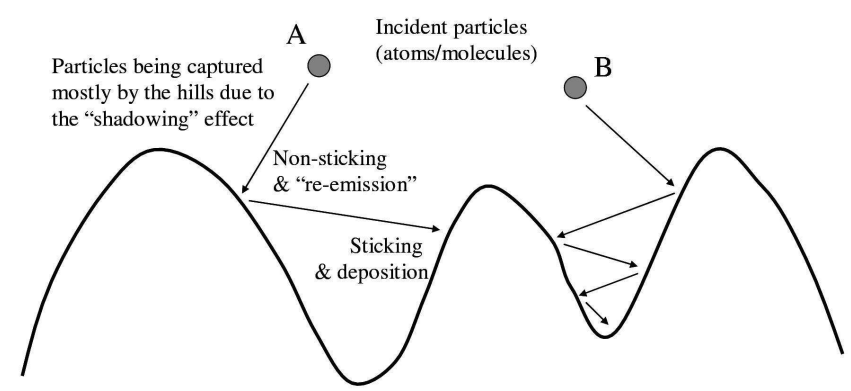

Figure 1: Surface of a growing thin film (growth front) under shadowing and re-emission effects.

We define a concise mapping between a network and the basic physical operations taking place in the growth process. We, then, develop qualitative and quantitative understanding of the growth dynamics by studying the corresponding network model. We present our initial results based on previously recorded simulations of the growth process.

The rest of the paper is organized as follows: We start with covering the thin film and nanostructure growth process and the basic physical effects involved in Section 2. We then survey the applications of dynamic network models on various areas in Section 3. Section 4 describes the details of our methodology of mapping growth dynamics to a network. We present initial results of our network-based modeling approach in Section 5, and conclude in Section 6.

\section{BASICS OF THIN FILM AND NANOS- TRUCTURE GROWTH}

Only recently, it has been recognized that in order to better explain the dynamics of surface growth one should considers the effects of both "shadowing" and "re-emission" processes [8]. As shown in Figure 1, particles can approach the surface at oblique angles and be captured by higher surface points (hills) due to the shadowing effect. This leads to the formation of rougher surfaces with columnar structures that can also be engineered to form "nanostructures" under extreme shadowing conditions, as in the case of oblique angle deposition that can produce arrays of nanorods and nanosprings [7]. In addition, depending on the detailed deposition process, particles can either stick to or bounce off from their impact points, which is determined by a sticking probability, also named "sticking coefficient" (s). Nonsticking particles are re-emitted and can arrive at other surface points including shadowed valleys. In other words, reemission has a smoothening effect while shadowing tries to roughen the surface. Both the shadowing and re-emission effects have been proven to be dominant over the surface diffusion and noise, and act as the main drivers of the dynamical surface growth front [7]. The prevailing effects of shadowing and re-emission rely on their "non-local" character: The growth of a given surface point depends on the heights of near and far-away surface locations due to shadowing and existence of re-emitted particles that can travel over long distances.

Due to the complexity of the shadowing and re-emission effects, no growth model has been developed yet within the framework of dynamical scaling theory that take into both these effects and still that can be analytically solved to predict the morphological evolution of thin film or nanostructure deposition. A dynamic growth equation that was pro- posed by Drotar et al. [4] and developed for plasma and reactive ion etching processes (where in etching surface atoms are removed instead of being incorporated to the surface as in the case of deposition) that take into the re-emission and shadowing effects could only be solved numerically for a limited case of re-emission and shadowing scenarios. Only recently, shadowing and re-emission effects could be fully incorporated into the Monte Carlo lattice simulation approaches $[9,8,4]$.

\section{DYNAMIC NETWORK MODELS}

The study of complex networks pervades various areas of science ranging from sociology to statistical physics [3]. A network in terms of modeling can be defined as a set nodes with links connecting them. Examples of real life complex networks include the Internet, the World Wide Web, metabolic networks, transportation networks, social networks, etc. Recent works, motivated by a large number of natural and artificial systems, such as the ones listed above, have turned the focus onto processes on networks, where the interaction and dynamics between the nodes are facilitated by a complex network. Here, our aim is to construct the network from the apparent dynamics.

By using network-based modeling, fundamental understanding of many natural and artificial systems has been attained. In complex networks research, two major types of network models are used for various applications: Smallworld [10] and scale-free (power-law) [1] networks. Watts and Strogatz, inspired by a sociological experiment, have proposed a network model known as the small-world (SW) network, which means that, despite their often large size, there is a relatively short path between any two nodes in most networks with some degree of randomness. The SW network was originally constructed as a model to interpolate between regular lattices and completely random networks. Systems and models (with well known behaviors on regular lattices) have been studied on SW networks, such as the Ising model, phase ordering, the Edwards-Wilkinson model, diffusion, and resistor networks.

The other major type of network is based on an observation made in the context of real networks such as the Internet, World Wide Web, scientific collaboration network, and e-mail network. The common characteristic among these networks is that they all exhibit power-law degree (connectivity) distributions. These networks are commonly known as power-law or scale-free networks [1] since their degree distributions are free of scale (i.e., not a function of the number of nodes $N$ ) and follow power-law distributions over many orders of magnitude. This phenomenon has been represented by the probability of having nodes with $\mathrm{k}$ degrees as $P(k) \sim k^{-\gamma}$ where $\gamma$ is usually between 2 and 3 . The origin of the scale-free behavior can be traced back to two mechanisms that are present in many systems, and have a strong impact on the final topology. First, networks are developed by the addition of new nodes that are connected to those already present in the system. This mechanism signifies continuous expansion in real networks. Second, there is a higher probability that a new node is linked to a node that already has a large number of connections. With appropriate mapping to a network model, both of these mechanisms can be qualitatively shown in thin film and nanostructure growth dynamics. If we consider the thin film surface as a set of nodes and re-emissions as the links between them, 

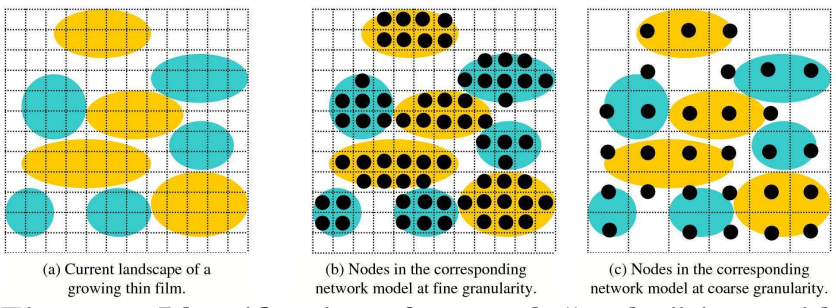

Figure 2: Identification of network "nodes" in a grid network model corresponding to a landscape of a growing thin film.

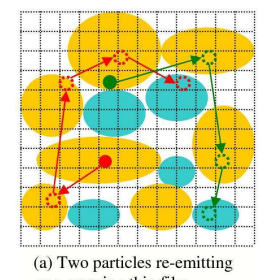

on a growing thin film

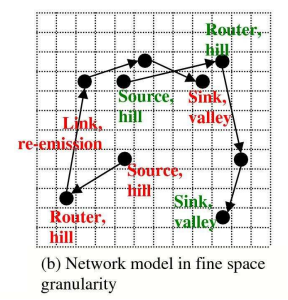

Figure 3: Grid network model development in space: Consider two, red and green, particles falling on a growing thin film sample. The red particle makes four re-emissions while the green one makes three re-emissions. We model each re-emission as a "link" between the nodes corresponding to the starting and ending points of the re-emission.

the first mechanism refers to the understanding that each particle gets "connected" to the grid network by falling on to the film surface. Similarly, the second mechanism refers to that a falling particle will more likely to land on a largesize node thereby contributing to the scale-free topological behavior of the growth dynamics.

\section{MAPPING GROWTH DYNAMICS TO A NETWORK MODEL}

Interestingly, non-local interactions among the surface points of a growing thin film that originate from shadowing and reemission effects can lead to non-random preferred trajectories of atoms/molecules before they finally stick and get deposited. For example, during re-emission, the path between two surface points where a particle bounces off from the first and head on to the second can define a "network link" between the two points. If the sticking coefficient is small, then the particle can go through multiple re-emissions that form links among many more other surface points. In addition, due to the shadowing effect, higher surface points act as the locations of first-capture and centers for re-emitting the particles to other places. In this manner, hills on a growing film resembles to the network "nodes" of heavy traffic, where the traffic is composed by the amount of re-emitted particles.

Several issues need to be considered in making a useful and appropriate mapping between the growth dynamics of thin films and nanostructures to a network modeling framework. Let us consider a snapshot of a growing thin film's landscape. In Figure 2(a), let us say that blue color shows currently elevated (i.e. hills) regions of the film and yellow color shows currently not elevated (i.e. valleys) regions of the film. The first mapping issue is to define a "node" in the corresponding network model. That is, what should be the boundary of the corresponding network node on the thin film surface? Intuitively, each blue or yellow region in

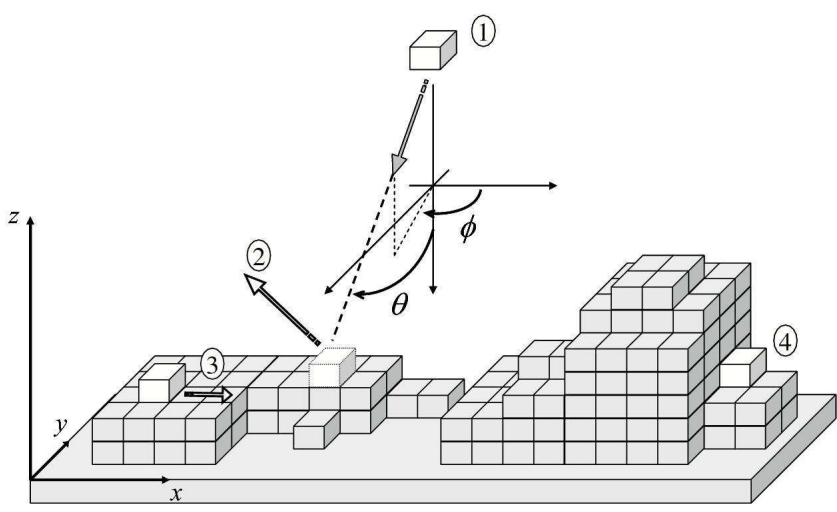

Figure 4: Some basic processes in the simulation: (1) A particle is sent towards surface with angles $\theta$ and $\phi$ based on an angular distribution chosen based on the deposition technique. This particle sticks to the surface with probability $s_{0}$. (2) If it does not stick, then it is re-emitted after which it may find another surface feature and stick there with probability $s_{1}$. This re-emission process continues like this for higher-order particles, too. (3) An adatom can diffuse on the surface. (4) Some surface points are shadowed from the incident and re-emission fluxes of particles due to the nearby higher surface features.

Figure 2(a) should ideally get mapped to a network node. However, this depends on the resolution of the grid being used for developing a network model. If the grid resolution is too fine, then a blue/yellow region of the film can correspond to multiple nodes as in Figure 2(b). Conversely, if the grid resolution is too coarse, then multiple blue/yellow regions can correspond to one network node as in Figure 2(c). Having finer grid is more likely to capture dynamics of the growth; therefore, we will develop our network models in as fine granularity as possible. For a fine granularity network model, it is always possible to aggregate the data pertaining to neighboring nodes and observe the behavior at coarser granularity. This is illustrated in Figure 3, where the grid network model can be developed at various scales in space.

After fixing the placement of nodes on the thin film, we then map growth dynamics to components of the corresponding grid network model as shown in Figure 3. In general, we argue that we can make an analogy that hills and valleys are nodes of the network system, but hills act as distributing centers, and valleys as gathering centers due to the shadowing and re-emission effects, respectively. The reemissions of particles can, then, be modeled as a "link" from the re-emission's starting node to the re-emission's ending node. The time it takes for the particle to reach to its new point can be considered as the link's "propagation delay", which implicitly expresses the distance between the starting and the ending nodes of the re-emission. It is even possible to consider the link's "capacity" as the highest possible number of particles that can simultaneously travel from the starting and the ending nodes of the re-emission, which is limited by the physical space corresponding to the link and average size of the re-emitting particles.

Since it is not possible to experimentally track the trajectories of re-emitted and deposited atoms during dynamic thin film growth, we will use Monte Carlo simulation approaches instead that were already shown to efficiently mimic the experimental processes and correctly predict the dy- 

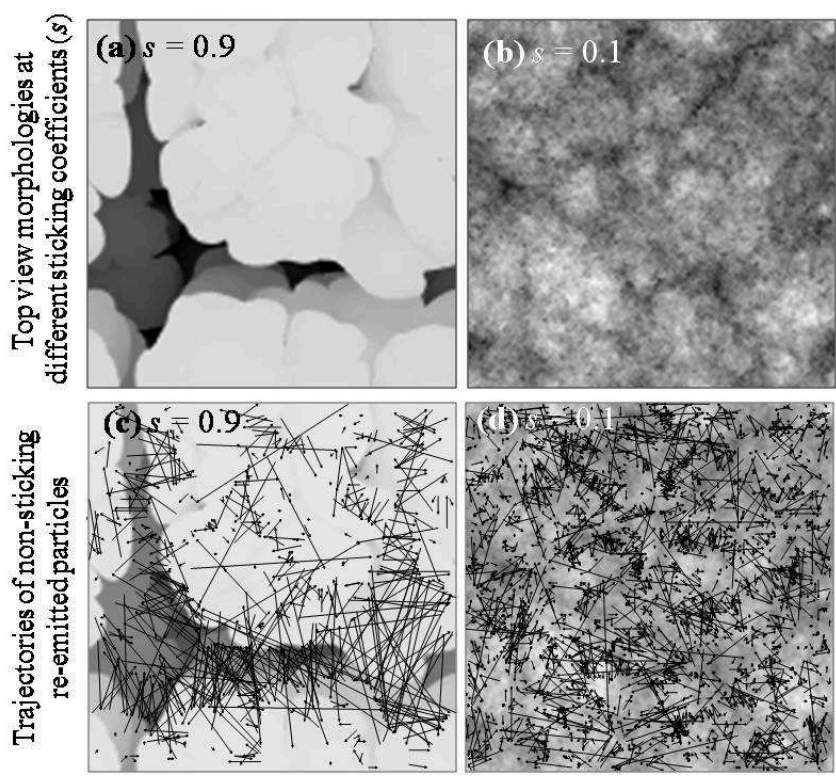

Figure 5: Top view images of simulated thin film surfaces grown under shadowing, re-emission, and noise effects for sticking coefficients (a) $s=0.9$ and (b) $s=0.1$. Corresponding projected trajectories of the re-emitted particles are also mapped on the top view morphologies for (c) $s=0.9$ and (d) $s=0.1$.

namic growth morphology. In these simulations, each incident particle (e.g., atom or molecule) is represented with the dimension of one lattice point. A specific angular distribution for the incident flux of particles is chosen depending on the deposition technique being simulated. At each simulation step, a particle is sent toward a randomly chosen lattice point on the substrate surface. Depending on the value of sticking coefficient $s$, the particle can bounce off and re-emit to other surface points. At each impact sticking coefficient can have different values represented as $s_{n}$, where $n$ is the order of re-emission ( $n=0$ being for the first impact $)^{1}$. In all the emission and re-emission processes shadowing effect is included, where the particle's trajectory can be cut-off by long surface features on its way to other surface points. After the incident particle is deposited onto the surface, it becomes a so called "adatom". Adatoms can hop on the surface according to some rules of energy, which is a process mimicking the surface diffusion. This simulation steps are repeated for other particles being sent onto the surface. Figure 4 illustrates the basic growth processes included in a typical Monte Carlo simulation approach.

\section{INITIAL RESULTS}

In order to explore existence of such a network behavior during thin film and columnar nanostructure growth, we developed 3D Monte Carlo simulations that take into shadowing, re-emission, surface diffusion, and noise effects. These effects simulate the evolution of surface topography and also the simulation environment allows us to record the trajectories of re-emitted atoms. As an example, Figure 5 shows the snapshot top view images of two surfaces simulated for a CVD type of deposition, at two different sticking coefficients.

\footnotetext{
${ }^{1}$ In this paper we assume a constant sticking coefficient for all subsequent re-emissions.
}

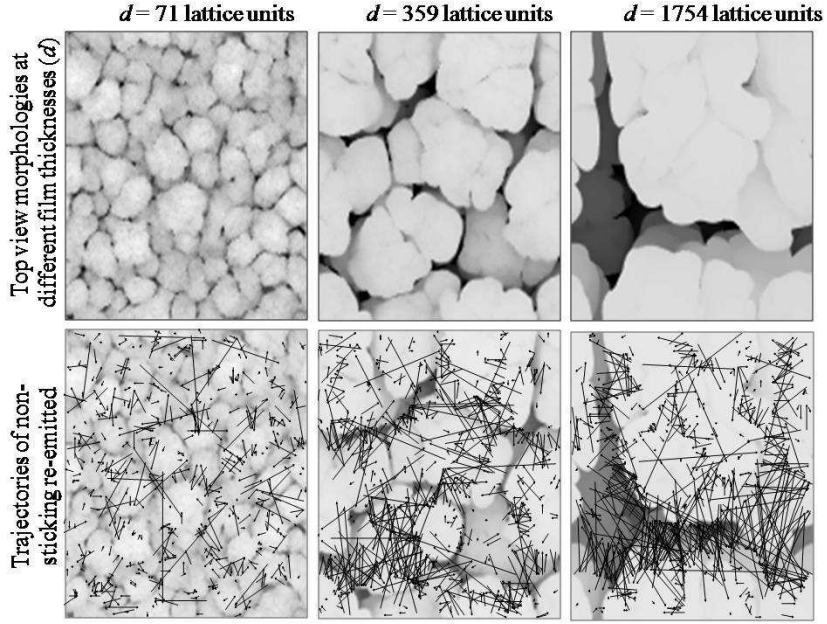

Figure 6: First row: Top view images from the simulated thin film surfaces for a CVD growth with $s=0.9$ at different film thicknesses $d$. Bottom row: Corresponding projected trajectories of the re-emitted particles qualitatively show the dynamic change in the network topography.

Figure 5 also displays their corresponding particle trajectories projected on the lateral plane. Qualitative network behavior can easily be realized in these simulated morphologies as the trajectories of re-emitted atoms "link" various surface points. It can also be seen that larger sticking coefficients (Figure 5(a) and Figure 5(c)) leads to fewer but longer range re-emissions, which are mainly among the peaks of columnar structures. Therefore, these higher surface points act as the "nodes" of the system. This is due to the shadowing effect where initial particles preferentially head on hills. They also have less chance to arrive down to valleys because of the high sticking probabilities (see also particle A illustrated in Figure 1). On the other hand, at lower sticking coefficients (Figure 5(b) and Figure 5(d)), particles now go through multiple re-emissions and can link many more surface points including the valleys that normally shadowed by higher surface points (e.g. particle B in Figure 1).

Another interesting observation revealed in our Monte Carlo simulations was the dynamic change of network behavior on the trajectories of re-emitted particles. Figure 6 shows top view images and their corresponding particle trajectories obtained from the simulations for a sticking coefficient of $s=0.9$, but this time at different film thicknesses that is proportional to the growth time. The dynamic change in the network topography can be clearly seen: at initial times, when the hills are smaller and more closely spaced, the re-emitted particles travel from one hill to another one or to a valley. However, as the film gets thicker, and some hills become higher than the others and get more separated, particles travel longer ranges typically among these growing hills. The shorter hills that get shadowed become the valleys of the system. It is expected that this dynamic behavior should be strongly dependent on the values of sticking coefficients and angular distribution of the incident flux of particles, which determine the strength of re-emission and shadowing effects, respectively. In other words, each deposition technique and material system can have different dynamic network behavior that can lead to various kinds of network systems. 


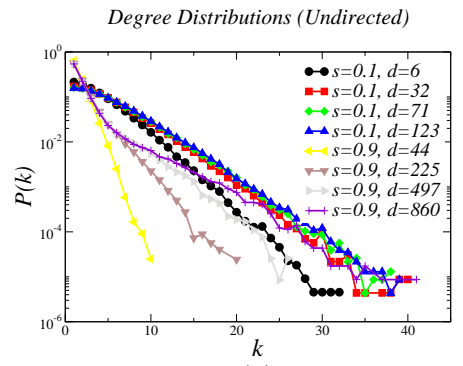

(a)

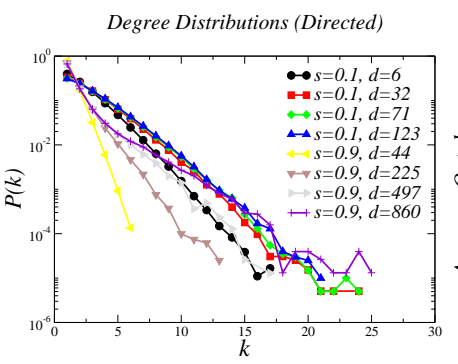

(b)

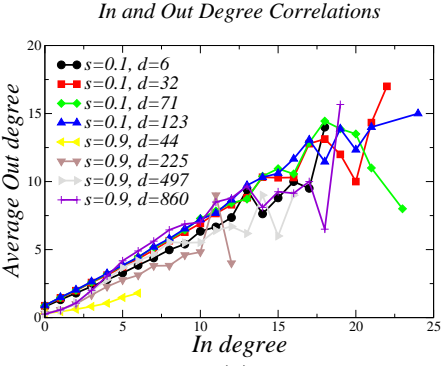

(c)

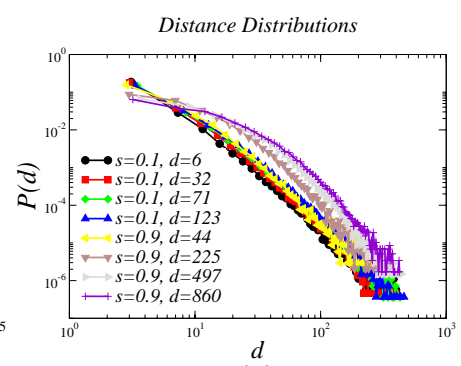

(d)

Figure 7: Behavior of degree and distance distributions for network models of a CVD thin film growth.

To make some initial observations on the network characteristics based on our network-based models of the growth dynamics, we plotted the degree and distance distributions in Figure 7 for a thin film of size $512 \times 512$ lattice units. We used each lattice unit on the thin film as a node in the corresponding network model and each re-emission as a directed/undirected link between the nodes of the surface locations. We developed the network models for snapshots of the growth where each snapshot being composed of $10 \times 512 \times 512$ particles' trajectories. We took four snapshots at different film thickness $d$. Since the complete growth process is very long this many particles, in some sense, samples the surface morphology. We did this network modeling for two different thin film growths, one with sticking coefficient $s=0.1$ and the other with $s=0.9$.

In this manner, Figure $7(\mathrm{a})$ and (b) shows the degree distribution for the network models of the snapshots when the links are undirected and directed respectively. Overall, the degree distributions exhibit an exponential behavior while becoming power-law as time progresses during the growth. This means that the interrelationship of the surface points become more dominant and some nodes (i.e., columnar structures) on the surface become the main hubs. The degree distributions are quite well characterized even though the growth dynamics are very chaotic. Another interesting observation is that, as time progresses, the degree distribution for the case with high $s$ converges to the one with low $s$, which is a non-intuitive result.

Figure 7(c) shows the relationship between the indegree and outdegree by plotting the average outdegree of nodes with a particular indegree value. From this graph also, it seems that the degree distributions converge to a common behavior as time progresses even though sticking coefficients are quire different. Similarly, Figure 7(d) shows the distance distribution of the links in the network, which clearly exhibits a power-law structure. The network model, again, clearly captures the behavior and shows that a higher sticking coefficient yields larger average distance with a pseudopower-law structure.

\section{CONCLUSIONS}

Our initial results on the observation of dynamic network behavior in simulated CVD thin films are very promising and indicate that a novel network modeling approach can be developed for various deposition systems. We showed that particles with non-unity sticking probabilities that are reemitted and deposited to other parts of the surface can form a network structure constructed by the links among each impact point, which defines nodes of the network. In addition, due to the shadowing effect where obliquely incident parti- cles hit preferentially to the higher surface points, hills of the morphology act as the hubs of the network where most of the particles are re-emitted from these regions. Columnar morphologies formed under high sticking coefficients promote the creation of long-distance network links mainly among the hills, while smoother morphologies of smaller sticking coefficient depositions leads to the formation of shorter range but well-connected links all over the surface points also including valleys. Therefore, this dynamic network behavior during thin film growth strongly depends on the sticking probabilities, presence of obliquely incident particles, and time-dependent morphology of the growing thin film, which leads to the realization of a rich dynamic network system. We believe that this work can lead to an unprecedented understanding of thin film and nanostructure growth, which has been long sought by the researchers.

\section{REFERENCES}

[1] A.-L. Barabási and R. Albert. Emergence of scaling in random networks. Science, 286:509, 1999.

[2] A.-L. Barabási and H. E. Stanley. Fractal Concepts in Surface Growth. Cambridge University Press, Cambridge, England, 1995.

[3] S. Boccaletti, V. Latora, Y. Moreno, M. Chavez, and D.-U. Hwang. Complex networks: Structure and dynamics. Physics Reports, 424:175, 2006.

[4] J. T. Drotar, Y.-P. Zhao, T.-M. Lu, and G.-C. Wang. Mechanisms for plasma and reactive ion etch-front roughening. Physical Review B, 61:3012, 2000.

[5] F. Family. Scaling of rough surfaces: effects of surface diffusion. Journal of Physics A, 19:L441, 1986.

[6] F. Family and T. Vicsek. Scaling of the active zone in the Eden process on percolation networks and the ballistic deposition model. Journal of Physics A, 18:L75, 1985.

[7] T. Karabacak and T.-M. Lu. Handbook of Theoretical and Computational Nanotechnology, chapter Shadowing growth and physical self-assembly of 3D columnar structures, page 729. American Scientific Publishers, Stevenson Ranch, CA, 2005.

[8] T. Karabacak, Y.-P. Zhao, G.-C. Wang, and T.-M. Lu. Growth front roughening in amorphous silicon films by sputtering. Physical Review B, 64:085323, 2001.

[9] T.-M. Lu, Y.-P. Zhao, J. T. Drotar, T. Karabacak, and G.-C. Wang. Novel mechanisms of the growth morphology thin films. In Proc. of the Material Research Society Symposium, volume 3, page 749, 2003.

[10] D. J. Watts and S. H. Strogatz. Collective dynamics of small-world networks. Nature, 393:440, 1998. 